\title{
Heterogeneity of ERBB2 in gastric carcinomas: a study of tissue microarray and matched primary and metastatic carcinomas
}

\author{
Eun Yoon Cho ${ }^{1,7}$, Kyeongmee Park ${ }^{2,7}$, Ingu Do ${ }^{3}$, Junhun Cho ${ }^{1}$, Jiyun Kim ${ }^{1}$, Jeeyun Lee ${ }^{4}$,
} Seonwoo Kim ${ }^{5}$, Kyoung-Mee Kim ${ }^{1}$, Tae Sung Sohn ${ }^{6}$, Won Ki Kang ${ }^{4}$ and Sung Kim ${ }^{6}$

${ }^{1}$ Department of Pathology, Samsung Medical Center, Sungkyunkwan University School of Medicine, Seoul, Korea; ${ }^{2}$ Department of Pathology, Inje University Sanggye Paik Hospital, Seoul, Korea; ${ }^{3}$ Samsung Cancer Research Institute, Sungkyunkwan University School of Medicine, Seoul, Korea; ${ }^{4}$ Department of Internal Medicine, Sungkyunkwan University School of Medicine, Seoul, Korea; ${ }^{5}$ Biostatistics Department, Samsung Biomedical Research Institute, Samsung Medical Center, Seoul, Korea and ${ }^{6}$ Department of Surgery, Samsung Medical Center, Sungkyunkwan University School of Medicine, Seoul, Korea

Trastuzumab in association with systemic cytotoxic chemotherapy is a therapeutic option for patients with advanced or metastatic ERBB2 + gastric carcinoma. The status of the ERBB2 overexpression or gene amplification is an important predictive marker in gastric cancer. However, it is controversial whether the primary tumor is representative of distant metastases in terms of ERBB2 status. Quadruplicated tissue microarrays from formalin-fixed paraffin-embedded tissues from 498 advanced primary gastric carcinomas and 97 matched metastatic lymph nodes were investigated by immunohistochemistry with HercepTest and silver in situ hybridization. For further comparison, another set of $\mathbf{4 1}$ paired primary and distant metastatic gastric carcinomas were also tested. Intratumoral heterogeneity was defined as different results between tissue microarray cores. ERBB2-positivity was observed in 52 gastric carcinomas (10\%) and was not associated with recurrence of disease or survival of patients. In ERBB2-positive primary gastric carcinomas, heterogeneous ERBB2 overexpression was observed in 21/63 (33\%) gastric carcinomas and heterogeneous ERBB2 gene amplification in 14/62 (23\%) cases. Repeated immunohistochemistry and silver in situ hybridization in representative paraffin tumor blocks confirmed focal ERBB2 overexpression and ERBB2 gene amplification and did not change the final results. Discrepancies in ERBB2 results between primary and paired metastatic lymph nodes were observed in $11 \%$ of cases by immunohistochemistry and $7 \%$ by silver in situ hybridization. Out of the 41 paired primary and distant metastases, $5(12 \%)$ cases were ERBB2-positive, and discrepancy was observed in one case. Intratumoral heterogeneity and discrepant ERBB2 results in primary and metastatic tumor are not uncommon in gastric carcinoma. Results of silver in situ hybridization showed less frequent heterogeneity compared with immunohistochemistry. Wherever possible, ERBB2 immunohistochemistry testing should be performed in both primary and distant metastatic sites.

Modern Pathology (2013) 26, 677-684; doi:10.1038/modpathol.2012.205; published online 14 December 2012

Keywords: Amplification; ERBB2; gastric; immunohistochemistry; metastatic; primary

The human protooncogene ERBB2 (commonly referred to as HER2) is a $185-\mathrm{kDa}$ transmembrane tyrosine kinase receptor and a member of the

Correspondence: Dr K-M Kim, M.D., Department of Pathology, Samsung Medical Center, Sungkyunkwan University School of Medicine, \#50 Ilwon-Dong, Kangnam-gu, Seoul 135-710, South Korea.

E-mail: kkmkys@skku.edu

${ }^{7}$ These authors contributed equally to this work.

Received 7 August 2012; revised 15 October 2012; accepted 15

October 2012; published online 14 December 2012 epidermal growth factor receptor family. ${ }^{1}$ Overexpression of ERBB2 has been found to promote tumorigenesis and is involved in the pathogenesis of several human cancers. Amplification of ERBB2 gene and overexpression of ERBB2 protein in gastric carcinoma were first described in 1986, and since then a number of studies have confirmed these findings. ${ }^{2}$

There is growing evidence that ERBB2 is an important biomarker and key driver of tumorigenesis in gastric cancer, showing amplification or overexpression in $7-53 \%$ of tumors. ${ }^{3-20} \mathrm{~A}$ recent 
multicenter phase III (ToGA) study showed a significant improvement in overall survival in patients with ERBB2 + advanced gastro-esophageal and gastric adenocarcinoma who were treated with chemotherapy plus trastuzumab compared with patients who received chemotherapy alone. ${ }^{3}$ Among various methods for measuring ERBB2 in carcinoma tissue, immunohistochemistry is the most attractive routine test based on issues of cost, convenience, and biological relevance.

Overexpression of ERBB2 protein highly correlates to amplification of the ERBB2 gene, ${ }^{21}$ and a significant discordance of ERBB2 status between the primary carcinoma and metastases has been reported in the breast. $^{22,23}$ However, the pattern seen in breast cancer has not yet been fully confirmed in gastric carcinoma. ${ }^{24}$ In the stomach, the frequency of immunohistochemistry $0 / 1+$ and fluorescence in situ hybridization (FISH)-positive gastric carcinoma is almost as high as that of immunohistochemistry $2+$ and FISH-positive samples (23\% vs 27\%), and ERBB2 expression, rather than ERBB2 amplification, is significantly correlated with longer overall survival. ${ }^{3}$ However, the prognostic significance of ERBB2 in gastric carcinoma is still limited and controversial. ${ }^{25}$

Heterogeneity of target expression within individual cancers and between primary tumors and metastases is an obstacle to determining eligibility for targeted therapy. ${ }^{20}$ In gastric carcinoma, ERBB2 heterogeneity has not been studied extensively. Marx et $a 1^{20}$ first reported homogeneous (not heterogeneous) amplification and expression of ERBB2 in primary gastric carcinomas and metastatic lymph nodes based on 69 paired one tissue microarray-cored gastric cancer samples. Recently, Kim et $a l^{26}$ suggested that the discordant ERBB2 amplification observed in metastatic lesions is explained substantially by genetic heterogeneity within primary tumors. Our previous study also showed that focal ERBB2 gene amplification is more common in gastric carcinomas with no $(0$ and $1+)$ or low $(2+)$ ERBB2 expression. ${ }^{27}$

To elucidate the intratumoral heterogeneity in primary and metastatic gastric carcinomas, we investigated overexpression and amplification of ERBB2 in 498 primary gastric carcinomas and 97 matched metastatic lymph nodes and an additional independent 41 paired primary and distant metastatic gastric carcinomas by immunohistochemistry and silver in situ hybridization.

\section{Materials and methods}

\section{Patients}

In total, 5133 patients with primary gastric adenocarcinoma underwent surgical treatment at Samsung Medical Center in Seoul, Korea from 2000 to 2004. Among them, 1048 patients with pT3 gastric carcinoma according to the seventh edition of the International Union Against Cancer classification were reviewed. ${ }^{28}$ Cases that underwent palliative surgery or preoperative chemotherapy, had a previous history of gastric surgery, or were diagnosed with multiple gastric cancers or any other malignancies were excluded. We retrieved the formalin-fixed, paraffinembedded blocks containing the primary tumor with/ without metastatic tumors of the lymph nodes from 498 patients, including $95 \mathrm{pN} 0,97 \mathrm{pN} 1,209 \mathrm{pN} 2$, and $97 \mathrm{pN} 3$ cases. All patients underwent radical gastrectomy with $\mathrm{D} 2$ lymph node dissection and adjuvant chemotherapy with 5-fluorouracil and leucovorin and were followed up regularly. The institutional review board approved this retrospective study.

Clinicopathological characteristics obtained from medical records included gender, age, tumor size, location, histological type, overall survival, and disease-free survival. Tumors were classified into two groups based on histology: (1) differentiated, which included papillary, well- or moderately differentiated adenocarcinoma, and well-differentiated mucinous carcinoma; and (2) undifferentiated, which included poorly or undifferentiated adenocarcinoma, signet ring cell carcinoma, and poorlydifferentiated mucinous carcinoma. The tumor recurrence patterns were evaluated in patients who underwent R0 resection and were classified as locoregional, peritoneal, distant, or combined recurrence. Clinicopathological variables were analyzed to determine their potential association with ERBB2 overexpression and ERBB2 gene amplification in gastric carcinoma tissues and metastatic lymph nodes.

To further investigate the discrepancies between primary gastric carcinoma and distant metastasis, an independent cohort consisting of 41 paired primary and distant metastasis specimens were retrieved between September 2007 and February 2012 and were also tested for ERBB2 overexpression and ERBB2 gene amplification. The sites of distant metastasis included the peritoneum $(n=11)$, liver $(n=10)$, colon $(n=6)$, lung or pleura $(n=4)$, ovary $(n=3)$, bone $(n=2)$, skeletal muscle $(n=2)$, rectal shelf $(n=1)$, larynx $(n=1)$, and axillary lymph node $(n=1)$. For this independent cohort with paired primary and distant metastatic tumors, as biopsy was preferred in most cases, whole-paraffin blocks were used for immunohistochemistry and silver in situ hybridization.

\section{Tissue Microarrays}

The surgical specimens were fixed in 10\% buffered formalin, processed, and embedded in paraffin using the standard protocol. All hematoxylin and eosin-stained slides were reviewed, and the representative areas were carefully selected and marked on all paraffin blocks. Four tissue cores $(0.6 \mathrm{~mm}$ diameter) were obtained separately from each primary carcinoma, and two tissue cores were sampled from each metastatic lymph node using an AccuMax (Isu Abxis Co., Ltd.). Four primary gastric carcinoma tissue cores were obtained from a single paraffin block, and a core was taken from each of the subserosal invasive front, both lateral sides, and the 
luminal surface area of the tumor. In cases with variable differentiation on hematoxylin and eosin staining, both differentiation areas were selected. For metastatic lymph nodes, $97 \mathrm{pN} 3$ cases were selected, with a mean number of metastatic lymph nodes of 17 (range 7-73), and tissue microarray cores were obtained from separate lymph nodes with massive extent of metastases.

\section{Immunohistochemistry}

ERBB2 protein expression was evaluated using the HercepTest kit (Dako, Glostrup, Denmark) according to the manufacturer's protocol. Tissue sections $(3 \mu \mathrm{m})$ were deparaffinized and rehydrated, and antigens were retrieved for $40 \mathrm{~min}$ in citrate buffer $\left(\mathrm{pH} \mathrm{6.1)}\right.$ at $95^{\circ} \mathrm{C}$. DAB was used as the chromogen, and the sections were counterstained with hematoxylin. Three independent pathologists (EC, KP, and KK) with no previous knowledge of clinical or pathological parameters, including silver in situ hybridization results, evaluated the staining data. ERBB2 immunoreactivity was scored according to ERBB2 overexpression assessment for biopsy specimens. ${ }^{3,29,30}$ In all cases with a $3+$ score, the three pathologists agreed perfectly, but in cases with a $2+$ score, there was disagreement. The final interpretation was determined by consensus. Intratumoral heterogeneity was defined as different results between tissue microarray cores. In cases with heterogeneity, a representative whole paraffin tumor block was tested to confirm the results.

\section{Silver In Situ Hybridization}

Consecutive unstained sections on electrostatically charged slides were prepared from the same tissue microarray paraffin blocks used for hematoxylin and eosin staining and silver in situ hybridization and were stained according to the manufacturer's protocols. Sequential in situ hybridization procedures for ERBB2 and CEN17 signal detection were conducted with the INFORM HER2 DNA, and CEN17 probes (Ventana Medical Systems, Tucson, AZ, USA) were performed using the Ventana ultraView SISH Detection Kit on a Ventana BenchMark XT automated slide stainer following the instructions provided by the manufacturer. In situ hybridization results were interpreted using the new guidelines for gastric ERBB2 by two observers (EC and ID). ${ }^{31}$

\section{Statistical Analysis}

SPSS 15.0 for Windows (SPSS, Chicago, IL, USA) was used to analyze the relationships between clinicopathological characteristics and the observed ERBB2 protein overexpression/ERBB2 gene amplification. The $\chi^{2}$ test and logistic regression analysis were used for univariate and multivariate analyses, respectively. Five-year overall survival was determined and compared between groups using the Kaplan-Meier method and log-rank test. Cox multivariate regression analysis was performed, and 95\% confidence intervals were determined. Results were considered significant when the $P$-values were $<0.05$.

\section{Results}

\section{Expression and Amplification of ERBB2 in Primary Gastric Carcinomas}

Among 498 primary gastric carcinomas, 52 cases $(10 \%)$ were immunohistochemistry $3+$ or $2+$ and silver in situ hybridization-positive. ERBB2 overexpression was found in 63/498 (13\%) primary gastric carcinomas and 16/97 (17\%) metastatic lymph nodes (Figure 1). ERBB2 gene amplification was observed in $62(12 \%)$ primary gastric carcinomas and $13(13 \%)$ metastatic lymph nodes.

Table 1 summarizes the clinicopathological findings observed in the gastric carcinomas with or without ERBB2 overexpression and ERBB2 gene amplification. ERBB2 overexpression and amplification was more common in male patients, older patients, Lauren intestinal type, and differentiated histology $(P<0.05)$. However, ERBB2-positivity was not associated with size or location of tumor, Borrmann gross type, lymphatic invasion, lymph node metastasis, recurrence of disease, or overall survival of patients $(P>0.05)$ (Figure 2). In a subanalysis of intestinal type gastric carcinomas by Lauren $(n=175)$, there was no significant difference in survival between the ERBB2-positive vs -negative groups.

Intratumoral heterogeneity of ERBB2 overexpression in primary gastric carcinoma was observed in $21 / 63(33 \%)$ gastric carcinomas, and heterogeneous ERBB2 gene amplification was observed in 14/62 $(23 \%)$ gastric carcinoma cases (Figure 3$)$. Repeated immunohistochemistry and silver in situ hybridization in representative paraffin tumor blocks confirmed focal ERBB2 overexpression and ERBB2 gene amplification and did not change the final results.

The concordance between immunohistochemistry and silver in situ hybridization was 95\%, and the correlation between immunohistochemistry and silver in situ hybridization results was statistically significant $(P<0.000001)$. Among 435 immunohistochemistry $0 / 1+$ cases, $11(3 \%)$ cases showed $E R B B 2$ gene amplification. Among 21 immunohistochemistry $2+$ cases, $10(48 \%)$ cases showed amplification of ERBB2 gene. All immunohistochemistry $3+$ gastric carcinomas showed amplification of $E R B B 2$ gene.

\section{Discrepancy of ERBB2 in Primary Tumors and Synchronous Metastatic Lymph Nodes}

ERBB2 overexpression was observed in 16/97 (17\%) metastatic lymph nodes and 17/97 (18\%) corresponding primary gastric carcinomas. ERBB2 gene amplification was found in 13/97 (13\%) metastatic 

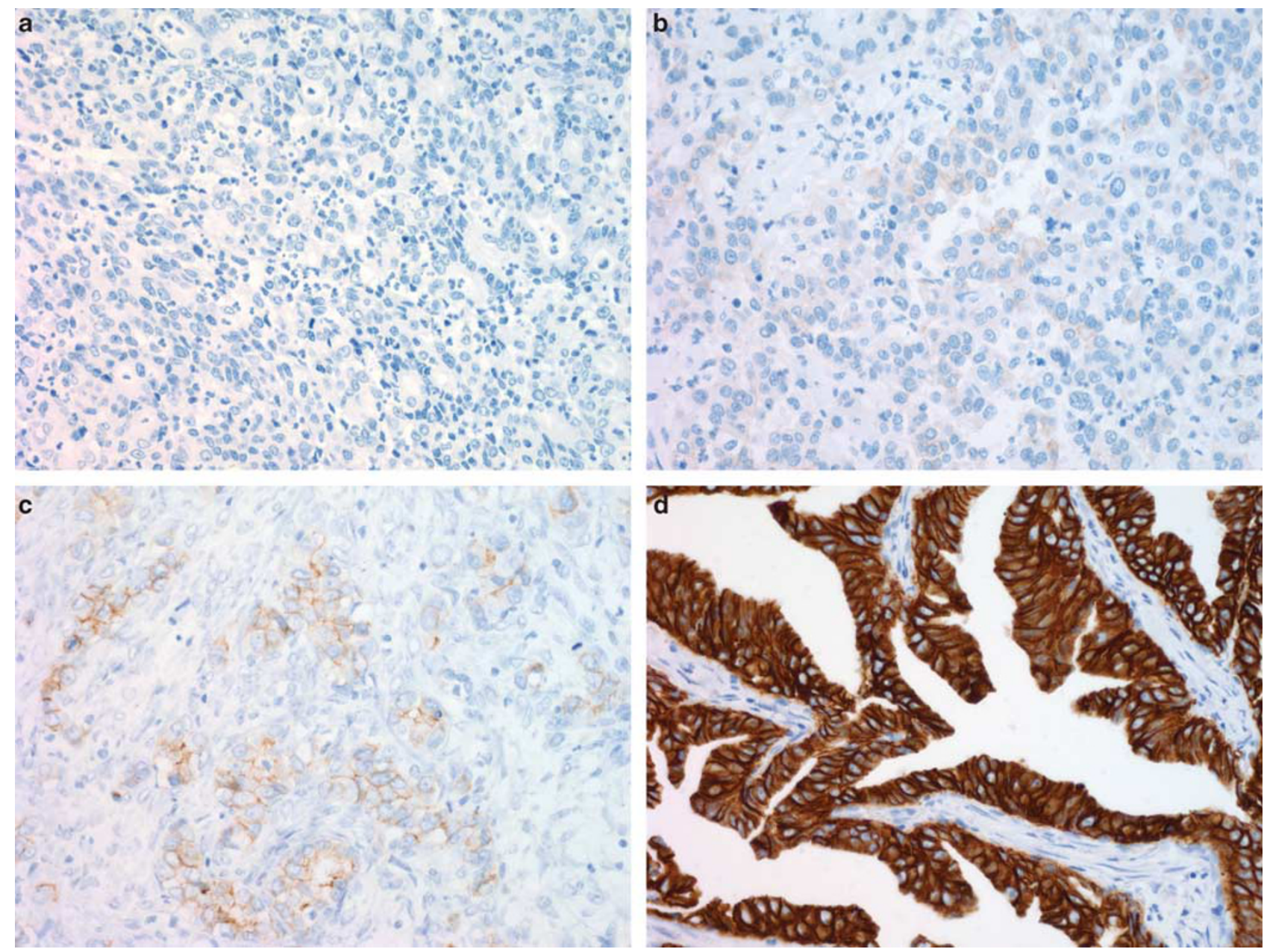

Figure 1 In immunohistochemistry, the expression of ERBB2 was interpreted and graded in accordance with the modified system. Scores of (a) 0 or (b) $1+$ were considered negative, (c) $2+$ was equivocally positive, and (d) $3+$ was strongly positive for ERBB2 overexpression.

lymph nodes and 16/97 (17\%) corresponding primary gastric carcinomas.

A discrepancy between primary gastric carcinomas and metastatic lymph nodes was noted in 11 $(11 \%)$ cases by immunohistochemistry and 7 (7\%) cases by silver in situ hybridization. In those discrepant cases, positive conversion (negative in the primary tumor and positive in the synchronous lymph node metastasis) was observed in $6 / 81(7 \%)$ cases by immunohistochemistry and 2/81 (3\%) cases by silver in situ hybridization. Likewise, negative conversion (positive in the primary tumor and negative in the lymph node metastasis) was observed in 5/16 (31\%) cases by immunohistochemistry and 5/16 (31\%) cases by silver in situ hybridization (Table 2).

\section{Heterogeneity of ERBB2 in Primary Gastric Carcinomas and Distant Metastases}

Of the 41 independent pairs of primary gastric tumors and distant metastases, $5(12 \%)$ primary tumors were ERBB2-positive by immunohistochemistry (4 ERBB2 $3+$ and 1 ERBB2 2+), and all of them showed ERBB2 gene amplification on silver in situ hybridization. In paired distant metastases, all ERBB2 $3+$ cases remained $3+$, and one case with $2+$ in immunohistochemistry and focal gene amplification of ERBB2 turned negative in distant metastasis.

\section{Discussion}

The purpose of this study was to assess intratumoral heterogeneity, discrepancies in ERBB2 status between primary gastric carcinoma and metastatic sites, and the role of ERBB2 in lymph node metastasis and clinical outcomes in patients with advanced gastric carcinoma. A therapeutically significant discordance in ERBB2 status between primary cancer and metastatic sites has been reported in the breast, urinary bladder, and prostate. $^{23,32,33}$ These differences could be due to possible genetic drift or clonal selection for ERBB2, which may happen during tumor progression, or to the presence of intratumoral heterogeneity of ERBB2. ${ }^{23,34}$ In the present study, a 
Table 1 Patient characteristics according to ERBB2 protein expression and amplification of ERBB2 gene

\begin{tabular}{|c|c|c|c|c|c|c|}
\hline \multirow{2}{*}{ Characteristics, n (\%) } & \multicolumn{3}{|c|}{ ERBB2 immunohistochemistry } & \multicolumn{3}{|c|}{ ERBB2 SISH } \\
\hline & $\begin{array}{l}\text { Positive } \\
(\mathrm{n}=63)\end{array}$ & $\begin{array}{l}\text { Negative } \\
(\mathrm{n}=435)\end{array}$ & $\mathrm{P}$-value & Amplified $(\mathrm{n}=62)(\%)$ & ) Not amplified $(\mathrm{n}=436)(\%)$ & $\mathrm{P}$-value \\
\hline Gender & & & 0.046 & & & 0.03 \\
\hline Male & $49(78)$ & $279(64)$ & & 49 (79) & $279(64)$ & \\
\hline Female & $14(22)$ & $156(36)$ & & $13(21)$ & $157(36)$ & \\
\hline Age (years) & $58.97 \pm 11.03$ & $53.53 \pm 11.87$ & 0.0007 & $58.60 \pm 11.13$ & $53.6 \pm 11.88$ & 0.0019 \\
\hline Tumor location & & & $0.072(0.85)^{\mathrm{a}}$ & & & $0.68(0.15)^{\mathrm{a}}$ \\
\hline Upper third & $9(14)$ & $70(16)$ & & $10(16)$ & $69(16)$ & \\
\hline Middle third & $17(27)$ & $183(42)$ & & $19(31)$ & $181(42)$ & \\
\hline Lower third & $32(51)$ & $153(35)$ & & $29(47)$ & $156(36)$ & \\
\hline Whole stomach & $5(8)$ & $29(7)$ & & $4(6)$ & $30(6)$ & \\
\hline Tumor size & $6.44 \pm 2.42$ & $6.39 \pm 3.08$ & 0.91 & $6.17 \pm 2.60$ & $6.43 \pm 3.06$ & 0.52 \\
\hline Type of operation & & & 0.81 & & & 0.9 \\
\hline Subtotal gastrectomy & $39(62)$ & $261(60)$ & & $37(60)$ & $263(60)$ & \\
\hline Total gastrectomy & $16(25)$ & $126(29)$ & & $17(27)$ & $125(29)$ & \\
\hline $\begin{array}{l}\text { Extended } \\
\text { gastrectomy }\end{array}$ & $8(13)$ & $48(11)$ & & $8(13)$ & $48(11)$ & \\
\hline Bormann type & & & 0.32 & & & 0.41 \\
\hline IV & $5(8)$ & $58(13)$ & & $5(8)$ & $55(13)$ & \\
\hline others & $58(92)$ & $380(87)$ & & $57(92)$ & $381(87)$ & \\
\hline Lauren classification & & & $<0.0001$ & & & $<0.0000001$ \\
\hline Diffuse & $13(20)$ & $281(65)$ & & $12(19)$ & $282(65)$ & \\
\hline Intestinal & $47(75)$ & $128(29)$ & & $47(76)$ & $128(29)$ & \\
\hline Mixed & $3(5)$ & $26(6)$ & & $3(5)$ & $26(6)$ & \\
\hline Histology & & & $<0.00001$ & & & $<0.0000001$ \\
\hline Differentiated & $45(71)$ & $122(28)$ & & $47(76)$ & $120(28)$ & \\
\hline Undifferentiated & $18(29)$ & $313(72)$ & & $15(24)$ & $316(72)$ & \\
\hline Lymphatic invasion & & & 0.89 & & & 0.79 \\
\hline Negative & $31(49)$ & $220(51)$ & & $30(48)$ & $221(51)$ & \\
\hline Positive & $32(51)$ & $215(49)$ & & $32(52)$ & $215(49)$ & \\
\hline$N$ stage & & & 0.35 & & & 0.5 \\
\hline No & $14(22)$ & $81(18)$ & & $11(18)$ & $84(19)$ & \\
\hline N1 & $10(16)$ & $87(20)$ & & $11(18)$ & $86(20)$ & \\
\hline N2 & $23(37)$ & $190(44)$ & & $24(39)$ & $189(43)$ & \\
\hline N3 & $16(25)$ & 77 (18) & & $16(25)$ & 77 (18) & \\
\hline Recurrence of disease & & & 0.29 & & & 0.72 \\
\hline Yes & $11(27)$ & $90(36)$ & & $12(32)$ & $89(36)$ & \\
\hline No & $30(73)$ & $158(64)$ & & $26(68)$ & $162(64)$ & \\
\hline Died of disease & & & 1 & & & 0.41 \\
\hline Yes & $28(44)$ & $195(45)$ & & $31(50)$ & $192(44)$ & \\
\hline No & $35(56)$ & $240(55)$ & & $31(50)$ & $244(56)$ & \\
\hline
\end{tabular}

${ }^{\mathrm{a} C o m p a r i s o n ~ b e t w e e n ~ u p p e r ~ t h i r d ~ v s ~ o t h e r ~ l o c a t i o n s . ~}$

discrepancy in ERBB2 between the primary gastric carcinoma and metastatic lymph node was found in $11 \%$ of cases by immunohistochemistry and in $7 \%$ by silver in situ hybridization. Furthermore, a discrepancy in ERBB2 status between paired primary gastric carcinoma and distant metastasis was also observed in one of the five ERBB2-positive cases. In our study, negative conversion was more frequently observed than positive conversion in metastatic lesions. However, positive conversion was more frequent than negative conversion (2.4 vs
$0.4 \%$ ) in a previous study. ${ }^{26}$ In those discrepant cases including ours, heterogeneous ERBB2 gene amplification was observed in primary tumor. These findings suggest that discordance in ERBB2 status between primary gastric cancer and metastatic sites may be caused by intratumoral heterogeneity of ERBB2 in primary tumor rather than genetic drift during tumor progression. These findings suggest that ERBB2 testing should be performed in both primary and metastatic sites whenever possible for trastuzumab therapy. 

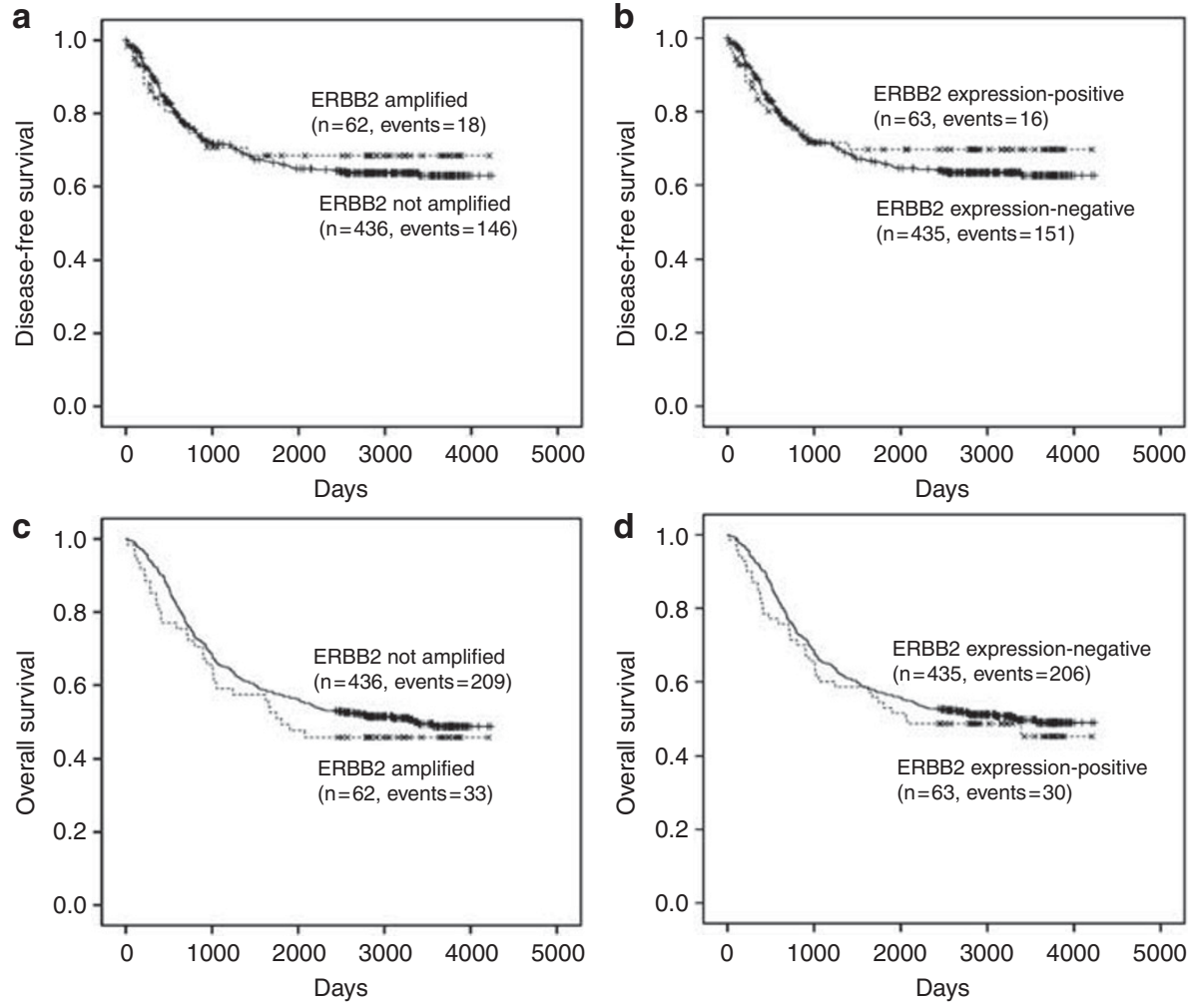

Figure 2 Disease-free survival curves of patients with pT3 gastric adenocarcinoma according to (a) ERBB2 amplification and (b) ERBB2 protein overexpression. Overall survival curves of patients with pT3 gastric adenocarcinoma according to (c) ERBB2 amplification and (d) ERBB2 overexpression.

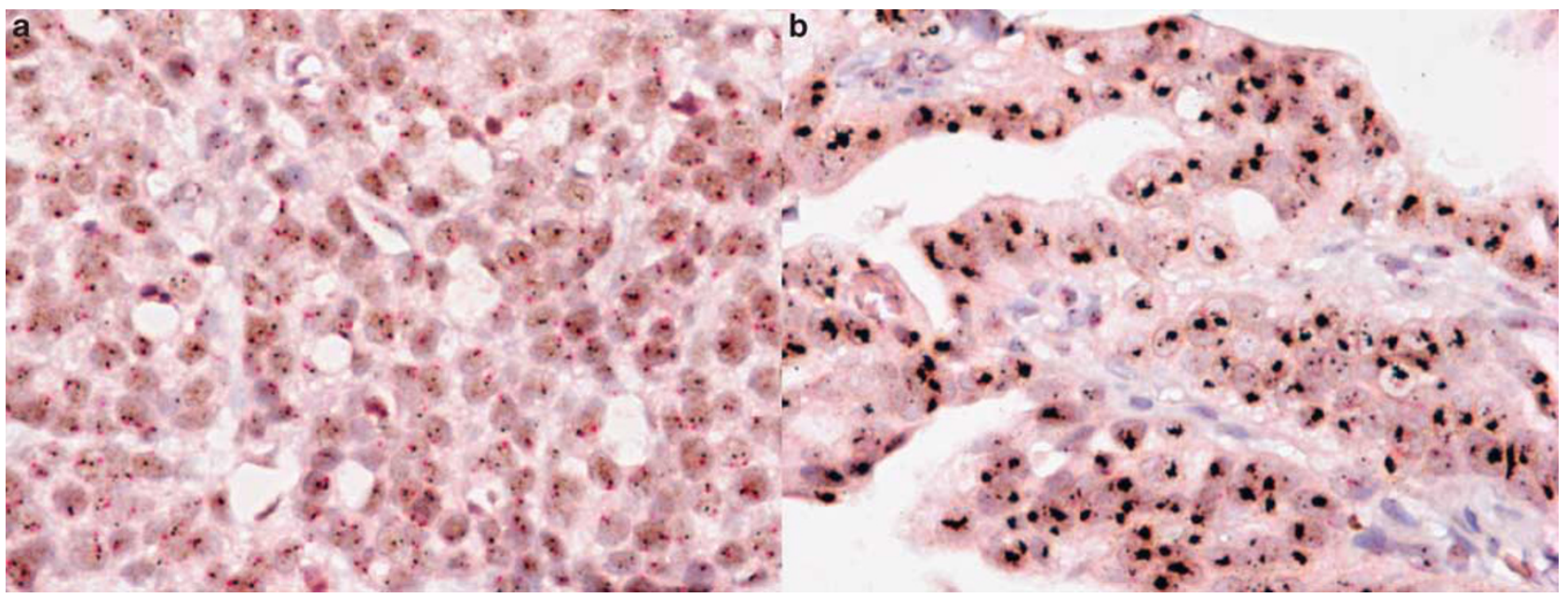

Figure 3 A representative photomicrograph of heterogeneous ERBB2 gene amplification determined by silver in situ hybridization with (a) poorly differentiated adenocarcinoma area without amplification and (b) moderately differentiated adenocarcinoma with amplification.

In previous studies, the overall frequency of ERBB2 overexpression in gastric cancer ranged from 7 to $53 \% .^{4-20}$ This wide range of overexpression of ERBB2 might be explained by the study cohort, localization within the stomach, histological subtype or interpretation of results. ERBB2 positivity is significantly higher in intestinal histology (34\%) and gastro-esophageal tumors (34\%) than in diffusetype $(6 \%)$ and gastric locations $(20 \%) .{ }^{25}$ In the present study, we used FDA-approved staining procedures and newly developed ERBB2 scoring criteria for gastric carcinoma, and our ERBB2positivity was similar to recently published large series with 2168 and 506 cases, respectively. ${ }^{3,35}$

The concordance of ERBB2 protein expression and gene amplification in gastric carcinomas is controversial. ${ }^{25}$ ERBB2 overexpression may occur through a number of different mechanisms, 
Table 2 Comparison of ERBB2 overexpression and ERBB2 gene amplification in primary gastric carcinomas and synchronous metastatic lymph nodes

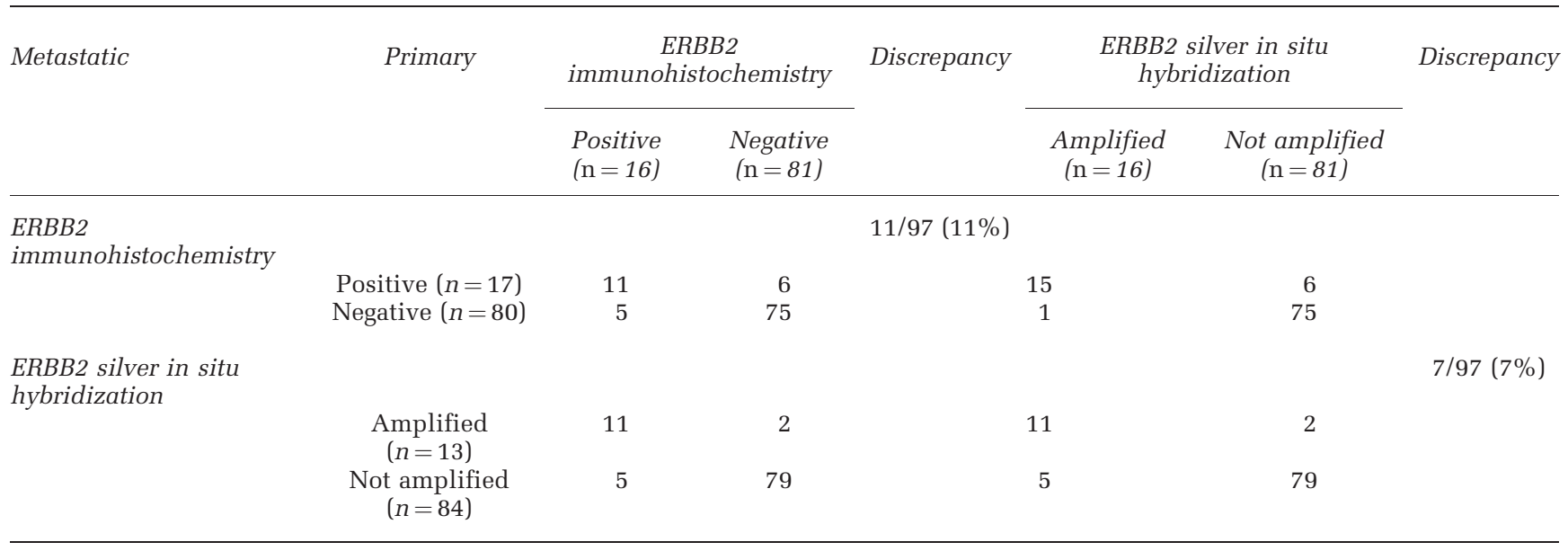

including transcriptional activation by other genes or post-transcriptional events. ${ }^{36}$ Recent studies have reported a high concordance between overexpression in immunohistochemistry and amplification by FISH. ${ }^{25}$ In the ToGA trial, the concordance between ERBB2 positivity detected by immunohistochemistry and FISH was $87 \%$, and the differences were largely due to FISH-positive cases that were immunohistochemistry $0 / 1+$, accounting for $22 \%$ of all ERBB2-positive cases. In our study, silver in situ hybridization-positive and immunohistochemistry $0 / 1+$ cases accounted for $3 \%$ of cases, and we achieved 95\% concordance between immunohistochemistry and silver in situ hybridization.

Marx et $a l^{20}$ reported that ERBB2 amplification is highly homogeneous in gastric carcinomas. Recently, Kim et $a l^{26}$ reported that the discordant ERBB2 amplification observed in metastatic lesions is explained substantially by genetic heterogeneity within primary tumors. In our study, we found intratumoral heterogeneity of ERBB2 expression in $4 \%$ and gene amplification in $3 \%$ of gastric carcinoma cases. In repeated immunohistochemistry and silver in situ hybridization performed on whole-paraffin tissue blocks, we found that these heterogeneities were really tumor inherent and not a sampling effect of tissue microarray, and these observations are similar to previous reports. ${ }^{29,37}$

Controversy remains as to whether ERBB2 is a prognostic factor in gastric cancer. Some studies have reported that ERBB2 is a poor prognostic factor in gastric carcinoma. ${ }^{4-18}$ However, Grabsch et al, ${ }^{35} \mathrm{Kim}$ et $a l,{ }^{37}$ and Barros-Silva et $a l^{38}$ reported that ERBB2 is not associated with prognosis in large series consisting of 924, 248, and 463 patients, respectively. The disagreement is probably related to differences in sample size, patient characteristics, and interpretation of results. ${ }^{35}$ Moreover, most studies have included both early and advanced gastric cancers in their study cohorts, so data concerning exclusively advanced disease are, for the most part, lacking. In this study of 498 patients with advanced gastric cancer, we found that ERBB2 overexpression or amplification was not associated with lymph node metastasis in pT3 gastric carcinomas and was not a significant prognostic factor.

In summary, intratumoral heterogeneity and discordant ERBB2 results in primary and metastatic tumor are not uncommon in gastric carcinoma. Results of silver in situ hybridization showed less frequent heterogeneity compared with immunohistochemistry. Wherever possible, ERBB2 immunohistochemistry testing should be performed in both primary and metastatic sites, and in cases with discrepancy, silver in situ hybridization test should be added.

\section{Acknowledgements}

This study was supported by a grant from the Korea Healthcare Technology R\&D Project, Ministry for Health and Welfare Affairs, Republic of Korea (A092255 and A101130) and a Samsung Biomedical Research Institute grant, \#SBRI-CB11031.

\section{Disclosure/conflict of interest}

The authors declare no conflict of interest.

\section{References}

1 Samanta A, LeVea CM, Dougall WC, et al. Ligand and p185c-neu density govern receptor interactions and tyrosine kinase activation. Proc Natl Acad Sci USA 1994;91:1711-1715.

2 Sakai K, Mori S, Kawamoto T, et al. Expression of epidermal growth factor receptors on normal human gastric epithelia and gastric carcinomas. J Natl Cancer Inst 1986;77:1047-1052.

3 Bang YJ, Van Cutsem E, Feyereislova A, et al. Trastuzumab in combination with chemotherapy versus chemotherapy alone for treatment of HER2-positive advanced gastric or gastro-oesophageal junction cancer 
(ToGA): a phase 3, open-label, randomised controlled trial. Lancet 2010;376:687-697.

4 Song Y, Huang J, Wang JW. Relationship between HER2/neu gene amplification and protein expression and prognosis in patients with advanced gastric carcinoma. Ai Zheng 2010;29:76-81.

5 Yonemura Y, Ninomiya I, Yamaguchi A, et al. Evaluation of immunoreactivity for erbB-2 protein as a marker of poor short term prognosis in gastric cancer. Cancer Res 1991;51:1034-1038.

6 Roh JK, Paik S, Chung HC, et al. Overexpression of erbB2 protein in gastric adenocarcinoma-a potential role in therapeutic response to adjuvant 5-FU-doxorubicin regimen. Gan To Kagaku Ryoho 1992;19:1207-1219.

7 Yonemura Y, Ninomiya I, Ohoyama S, et al. Correlation of c-erbB-2 protein expression and lymph node status in early gastric cancer. Oncology 1992;49:363-367.

8 Uchino S, Tsuda H, Maruyama K, et al. Overexpression of c-erbB-2 protein in gastric cancer. Its correlation with long-term survival of patients. Cancer 1993;72:3179-3184.

9 Lee HR, Kim JH, Uhm HD, et al. Overexpression of c-ErbB-2 protein in gastric cancer by immunohistochemical stain. Oncology 1996;53:192-197.

10 Shun CT, Wu MS, Lin JT, et al. Relationship of p53 and c-erbB-2 expression to histopathological features, Helicobacter pylori infection and prognosis in gastric cancer. Hepatogastroenterology 1997;44:604-609.

$11 \mathrm{Wu}$ MS, Shun CT, Wang HP, et al. Genetic alterations in gastric cancer: relation to histological subtypes, tumor stage, and Helicobacter pylori infection. Gastroenterology 1997;112:1457-1465.

12 Allgayer H, Babic R, Gruetzner KU, et al. c-erbB-2 is of independent prognostic relevance in gastric cancer and is associated with the expression of tumor-associated protease systems. J Clin Oncol 2000;18:2201-2209.

13 Sanz-Ortega J, Steinberg SM, Moro E, et al. Comparative study of tumor angiogenesis and immunohistochemistry for p53, c-ErbB2, c-myc and EGFr as prognostic factors in gastric cancer. Histol Histopathol 2000;15:455-462.

14 Wang YL, Sheu BS, Yang HB, et al. Overexpression of C-erb-B2 proteins in tumor and non-tumor parts of gastric adenocarcinoma-emphasis on its relation to H. pylori infection and clinicohistological characteristics. Hepatogastroenterology 2002;49:1172-1176.

15 Takehana T, Kunitomo K, Kono K, et al. Status of c-erbB-2 in gastric adenocarcinoma: a comparative study of immunohistochemistry, fluorescence in situ hybridization and enzyme-linked immuno-sorbent assay. Int J Cancer 2002;98:833-837.

16 Pinto-de-Sousa J, David L, Almeida R, et al. c-erb B-2 expression is associated with tumor location and venous invasion and influences survival of patients with gastric carcinoma. Int J Surg Pathol 2002;10:247-256.

17 Park DI, Yun JW, Park JH, et al. HER-2/neu amplification is an independent prognostic factor in gastric cancer. Dig Dis Sci 2006;51:1371-1379.

18 Zhang XL, Yang YS, Xu DP, et al. Comparative study on overexpression of HER2/neu and HER3 in gastric cancer. World J Surg 2009;33:2112-2118.

19 Yu GZ, Chen Y, Wang JJ. Overexpression of Grb2/HER2 signaling in Chinese gastric cancer: their relationship with clinicopathological parameters and prognostic significance. J Cancer Res Clin Oncol 2009;135:1331-1339.

20 Marx AH, Tharun L, Muth J, et al. HER-2 amplification is highly homogenous in gastric cancer. Hum Pathol 2009;40:769-777.
21 Hoang MP, Sahin AA, Ordonez NG, et al. HER-2/neu gene amplification compared with HER-2/neu protein overexpression and interobserver reproducibility in invasive breast carcinoma. Am J Clin Pathol 2000;113:852-859.

22 Tapia C, Savic S, Wagner U, et al. HER2 gene status in primary breast cancers and matched distant metastases. Breast Cancer Res 9:R31 2007.

23 Santinelli A, Pisa E, Stramazzotti D, et al. HER-2 status discrepancy between primary breast cancer and metastatic sites. Impact on target therapy. Int J Cancer 2008; 122:999-1004.

24 Jorgensen JT. Targeted HER2 treatment in advanced gastric cancer. Oncology 2010;78:26-33.

25 Gravalos C, Jimeno A. HER2 in gastric cancer: a new prognostic factor and a novel therapeutic target. Ann Oncol 2008;19:1523-1529.

$26 \mathrm{Kim}$ MA, Lee HJ, Yang HK, et al. Heterogeneous amplification of ERBB2 in primary lesions is responsible for the discordant ERBB2 status of primary and metastatic lesions in gastric carcinoma. Histopathology 2011;59:822-831.

27 Cho EY, Srivastava A, Park K, et al. Comparison of four immunohistochemical tests and FISH for measuring HER2 expression in gastric carcinomas. Pathology 2012;44:216-220.

28 Sobin LGM, Wittekind C eds. TNM Classification of Malignant Tumours 7th edn. International Union Against Cancer (UICC): New York, Wiley, 2009.

29 Hofmann M, Stoss O, Shi D, et al. Assessment of a HER2 scoring system for gastric cancer: results from a validation study. Histopathology 2008;52:797-805.

30 Ruschoff J, Dietel M, Baretton G, et al. HER2 diagnostics in gastric cancer-guideline validation and development of standardized immunohistochemical testing. Virchows Arch 2010;457:299-307.

31 Ruschoff J, Hanna W, Bilous M, et al. HER2 testing in gastric cancer: a practical approach. Mod Pathol 2012;25:637-650.

32 Morris MJ, Reuter VE, Kelly WK, et al. HER-2 profiling and targeting in prostate carcinoma. Cancer 2002; 94:980-986.

33 Jimenez RE, Hussain M, Bianco FJ Jr., et al. Her-2/neu overexpression in muscle-invasive urothelial carcinoma of the bladder: prognostic significance and comparative analysis in primary and metastatic tumors. Clin Cancer Res 2001;7:2440-2447.

34 Edgerton SM, Moore D 2nd, Merkel D, et al. erbB-2 (HER-2) and breast cancer progression. Appl Immunohistochem Mol Morphol 2003;11:214-221.

35 Grabsch H, Sivakumar S, Gray S, et al. HER2 expression in gastric cancer: Rare, heterogeneous and of no prognostic value - conclusions from 924 cases of two independent series. Cell Oncol 2010;32:57-65.

36 Kameda T, Yasui W, Yoshida K, et al. Expression of ERBB2 in human gastric carcinomas: relationship between p185ERBB2 expression and the gene amplification. Cancer Res 1990;50:8002-8009.

37 Kim MA, Jung EJ, Lee HS, et al. Evaluation of HER-2 gene status in gastric carcinoma using immunohistochemistry, fluorescence in situ hybridization, and realtime quantitative polymerase chain reaction. Hum Pathol 2007;38:1386-1393.

38 Barros-Silva JD, Leitao D, Afonso L, et al. Association of ERBB2 gene status with histopathological parameters and disease-specific survival in gastric carcinoma patients. Br J Cancer 2009;100:487-493. 